\title{
Sneutrino as Dark Matter candidate in an inverse see-saw scenario
}

\section{Federica Bazzocchi ${ }^{* \dagger}$}

AHEP Group, Institut de Física Corpuscular - C.S.I.C./Universitat de València

Edificio Institutos de Paterna, Apt 22085, E-46071 Valencia, Spain

E-mail: fbazzo@ific.uv.es

\begin{abstract}
We show how inverse seesaw minimal supergravity offers a common solution to the generation of the neutrino mass and to the origin of dark matter. Such schemes naturally reconcile the small neutrino masses with the correct relic sneutrino dark matter abundance and accessible direct detection rates in nuclear recoil experiments.
\end{abstract}

Identification of dark matter 2008

August 18-22, 2008

Stockholm, Sweden

\footnotetext{
* Speaker.

${ }^{\dagger}$ Based on C. Arina, F. Bazzocchi, N. Fornengo, J.C. Romao, J.W.F. Valle, Phys.Rev.Lett.101:161802,2008
} 


\section{Introduction}

Cosmological studies clearly show that a large fraction of the mass of the Universe is dark and must be non-baryonic. Extension of the Standard Model (SM) provides a huge number of possible dark matter candidates, both hot, warm and cold. Among the different candidates, a large number arises by minimal or non minimal supersymmetric extensions of the Standard Model, such the neutralino, the gravitino and the axino. In the Minimal Supersymmetric Syandard Model (MSSM) the sneutrino can not be the cold dark matter (CDM) candidate ([1] and references therein). However it has been shown that non minimal SUSY scenario can produce a sneutrino cold dark matter candidate [7], [?]. . In particular [7] provides a further scenario in which the generation of neutrino masses may provide new insight on the nature of the dark matter [3]. Indeed it has been shown that once we add to the normal MSSM the singlet superfields associated to the right-handed neutrinos responsible of the generation of neutrino masses through the normal see-saw mechanism, the corresponding neutrino superpartner, the sneutrino, can be a good CDM candidate. Crucial ingredients in the scenario described in [7] were a see-saw scale around $1 \mathrm{TeV}$ and no minimal supergravity (mSUGRA) universality conditions.

On the contrary, here we show that in a mSUGRA scheme where the smallness of neutrino masses is accounted for within the inverse seesaw mechanism the lightest supersymmetric particle is likely to be represented by the sneutrino instead of the lightest neutralino, opening a new window for the mSUGRA scenario.

In the following sections we briefly describe the model and demonstrate that such a model naturally reconciles the small neutrino masses with the correct relic abundance of sneutrino dark matter and experimentally accessible direct detection rates.

\section{Minimal SUGRA inverse seesaw model}

The Minimal SUGRA inverse seesaw model is obtained by adding to the MSSM three sequential pairs of $S U(2) \times U(1)$ singlet neutrino superfields $\hat{v}_{i}^{c}$ and $\hat{S}_{i}(i$ is the generation index), with the following superpotential terms $[4,5]$ :

$$
\mathscr{W}=\mathscr{W}_{\mathrm{MSSM}}+\varepsilon_{a b} h_{v}^{i j} \widehat{L}_{i}^{a} \widehat{v}_{j}^{c} \widehat{H}_{u}^{b}+M_{R}^{i j} \widehat{v}_{i}^{c} \widehat{S}_{j}+\frac{1}{2} \mu_{S}^{i j} \widehat{S}_{i} \widehat{S}_{j}
$$

where $\mathscr{W}_{\text {MSSM }}$ is the usual MSSM superpotential. In the limit $\mu_{S}^{i j} \rightarrow 0$ there are exactly conserved lepton numbers assigned as $(1,-1,1)[4,5]$ for $v, v^{c}$ and $S$, respectively.

The SUSY-breaking Lagrangian is given by adding to the MSSM SUSY-breaking Lagrangian $\mathscr{L}_{\text {soft }}^{\text {MSSM }}$ the new soft-breaking terms related to the additional singlet fields

$$
\begin{aligned}
-\mathscr{L}_{\mathrm{soft}}= & -\mathscr{L}_{\mathrm{soft}}^{\mathrm{MSSM}}+\tilde{v}_{i}^{c} \mathbf{M}_{v^{c} i j}^{2} \tilde{v}_{j}^{c}+\tilde{S}_{i} \mathbf{M}_{\mathbf{S}_{i j}}^{2} \tilde{S}_{j} \\
& +\varepsilon_{a b} A_{h_{v}}^{i j} \tilde{L}_{i}^{a} \tilde{v}_{j}^{c} H_{u}^{b}+B_{M_{R}}^{i j} \tilde{v}_{i}^{c} \tilde{S}_{j}+\frac{1}{2} B_{\hat{\mu}_{S}}^{i j} \tilde{S}_{i} \tilde{S}_{j} .
\end{aligned}
$$

Neutrino masses are generated through the inverse seesaw mechanism $[4,5,6]$ : the effective neutrino mass matrix $m_{v}^{\text {eff }}$ is obtained by the following relation:

$$
m_{v}^{\text {eff }}=-v_{u}^{2} h_{v}\left(M_{R}^{T}\right)^{-1} \mu_{S} M_{R}^{-1} h_{v}^{T}=\left(U^{T}\right)^{-1} m_{\mu}^{\text {diag }} U^{-1}
$$


with $h_{v}$ the Yukawa matrix and $v_{u}$ the $H_{u}$ vacuum expectation value. The smallness of the neutrino mass is related to the smallness of the $\mu_{S}$ parameter. Light neutrino masses around the $\mathrm{eV}-$ or smaller- allow for a sizeable magnitude for the Dirac-type mass $m_{D}=v_{u} h_{v}$ around $100 \mathrm{GeV}$ and a Dirac-type mass $M_{R}$ around the TeV.

The main feature of the model is that the nature of the dark matter and the generation of neutrino masses arise from the same sector. In order to illustrate the mechanism we consider the simplest one-generation case. In this case the sneutrino mass matrix reads:

$$
\mathscr{M}^{2}=\left(\begin{array}{cc}
\mathscr{M}_{+}^{2} & \mathbf{0} \\
\mathbf{0} & \mathscr{M}_{-}^{2}
\end{array}\right)
$$

where

$$
\mathscr{M}_{ \pm}^{2}=\left(\begin{array}{ccc}
m_{L}^{2}+\frac{1}{2} m_{Z}^{2} \cos 2 \beta+m_{D}^{2} & \pm\left(A_{h_{v}} v_{u}-\mu m_{D} \operatorname{cotg} \beta\right) & m_{D} M_{R} \\
\pm\left(A_{h_{v}} v_{u}-\mu m_{D} \operatorname{cotg} \beta\right) & m_{v^{c}}^{2}+M_{R}^{2}+m_{D}^{2} & \mu_{S} M_{R} \pm B_{M_{R}} \\
m_{D} M_{R} & \mu_{S} M_{R} \pm B_{M_{R}} & m_{S}^{2}+\mu_{S}^{2}+M_{R}^{2} \pm B_{\mu_{S}}
\end{array}\right)
$$

in the CP eigenstates basis: $\Phi^{\dagger}=\left(\tilde{v}_{+}, \tilde{v}_{+}^{c}, \tilde{S}_{+}, \tilde{v}_{-}, \tilde{v}_{-}^{c}, \tilde{S}_{-}\right)$. Once diagonalized, the lightest of the six mass eigenstates is our dark matter candidate, and it is stable by $R$-parity conservation.

\section{Sneutrino LSP and Cold Dark Matter}

Let us now consider the model within a mSUGRA scenario. In the absence of the singlet neutrino superfields, the mSUGRA framework predicts the lightest supersymmetric particle (LSP) to be either a stau or a neutralino, and only the latter case represents a viable dark matter candidate, but WMAP bound considerably restrictes the cosmologically acceptable regions of parameter space [8].

In contrast, when the singlet neutrino superfields are added, a combination of sneutrinos emerges quite naturally as the LSP. Indeed, we have computed the resulting supersymmetric particle spectrum and couplings by adapting the SPheno code [9] so as to include the additional singlet superfields. An illustrative example of how the minimal SUGRA particle spectrum is modified by the presence of such states is given in Fig. 2.

A more general analysis in the mSUGRA parameter space is shown in the right panel of Fig. 2: the dark (blue) shaded area is excluded either by experimental bounds on supersymmetry and Higgs boson searches, or because it does not lead to electroweak symmetry breaking, while the (light) yellow region refers to stau LSP in the conventional (unextended) mSUGRA case. As expected, in all of the remaining region of the plane, the neutralino is the LSP in the standard mSUGRA case. The new phenomenological possibility which opens up thanks to the presence of the singlet neutrino superfields where the sneutrino is the LSP corresponds to the full dashed (red) and light (yellow) areas.

The novelty of the spectrum implied by mSUGRA implemented with the inverse seesaw mechanism is that it may lead to a bosonic dark matter candidate, the lightest sneutrino $\tilde{v}_{1}$, instead of the fermionic neutralino. To understand the physics it suffices for us to consider the simple one 

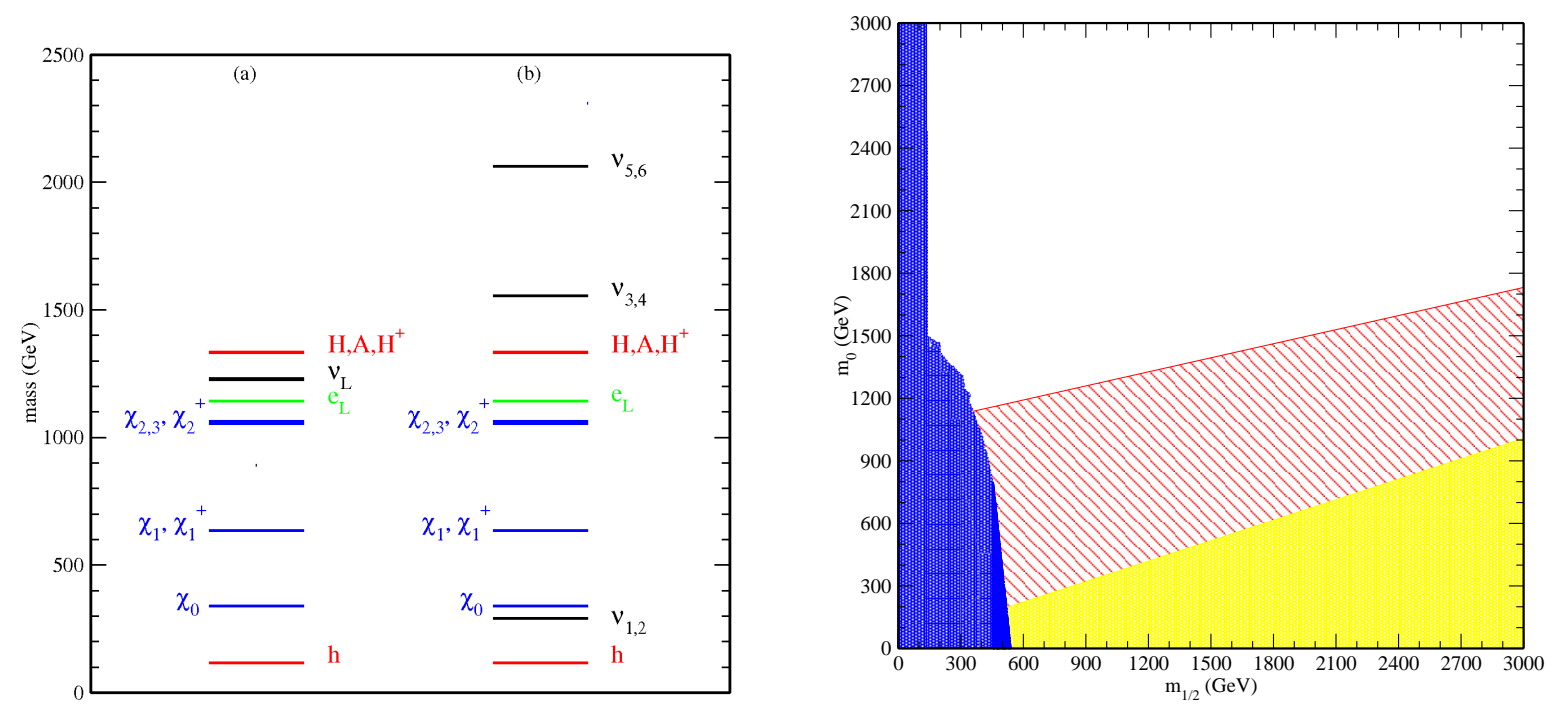

Figure 1: (left panel) Supersymmetric particle spectrum in the standard mSUGRA scheme [panel (a)] and in the inverse seesaw mSUGRA model [panel (b)] with parameters chosen as: $m_{0}=358 \mathrm{GeV}, m_{1 / 2}=692$ $\mathrm{GeV}, A_{0}=0, \tan \beta=35$ and sign $\mu>0$. The sneutrino sector has the additional parameter $B_{\mu_{S}}$, fixed at 10 $\mathrm{GeV}^{2}$. The squark sector is not shown.

Figure 2: (right panel) The $m_{0}-m_{1 / 2}$ plane for $\tan \beta=35, A_{0}=0$ and $\mu>0$. The shaded (red) and light (yellow) areas denote the set of supersymmetric parameters where the sneutrino is the LSP in inverse seesaw models (it includes all the light (yellow) region where the $\tilde{\tau}$ is the LSP in the standard mSUGRA case). The white region has the neutralino as LSP in both standard and modified mSUGRA. For the sneutrino LSP region, the additional parameters are: $B_{\mu_{S}}=10 \mathrm{GeV}^{2}, M_{R}=500 \mathrm{GeV}, m_{D}=5 \mathrm{GeV}$ and $\mu_{S}=100 \mathrm{eV}$. The dark (blue) region is excluded by experimental and theoretical constraints.

sneutrino generation case. For a detailed discussion on the relic density calculation for sneutrino dark matter, see Ref. [7]. From the left panel of Fig. 3 we see that a large fraction of the sneutrino configuration are compatible with the WMAP cold dark matter range, and therefore represents viable sneutrino dark matter models. The right panel of Fig. 3 in addition shows that direct detection experiments do not exclude this possibility: instead, a large fraction of configurations are actually compatible and under exploration by current direct dark matter detection experiments.

We stress that all models reported in Figs. 3 have the inverse seesaw-induced neutrino masses consistent with current experimental observations for natural values of its relevant parameters. smaller than about $80 \mathrm{GeV}^{2}$. The presence of the new scalar superfield $\hat{S}$ therefore plays a crucial role in controlling both the neutrino mass generation (through the parameter $\mu_{s}$ ) and the sneutrino relic abundance and direct detection cross section (through the parameter $B_{\mu_{S}}$ ). Note that our proposed scheme may also have important implications for supersymmetric particle searches at the LHC. Unfortunately being the neutralino the NNLSP (Fig. 2) many possible LHC signatures are expected to be almost indistinguishable by the corresponding MSSM ones. However in some cases it should be possible identify the inverse MSSM due to modified particle spectra and decay chains.

In conclusion, in this talk we have presented an mSUGRA scenario in which neutrino masses, generated by means of an inverse seesaw mechanism, and dark matter arise from the same sector of the theory. Over large portions of the parameter space the model successfully accommodates light 

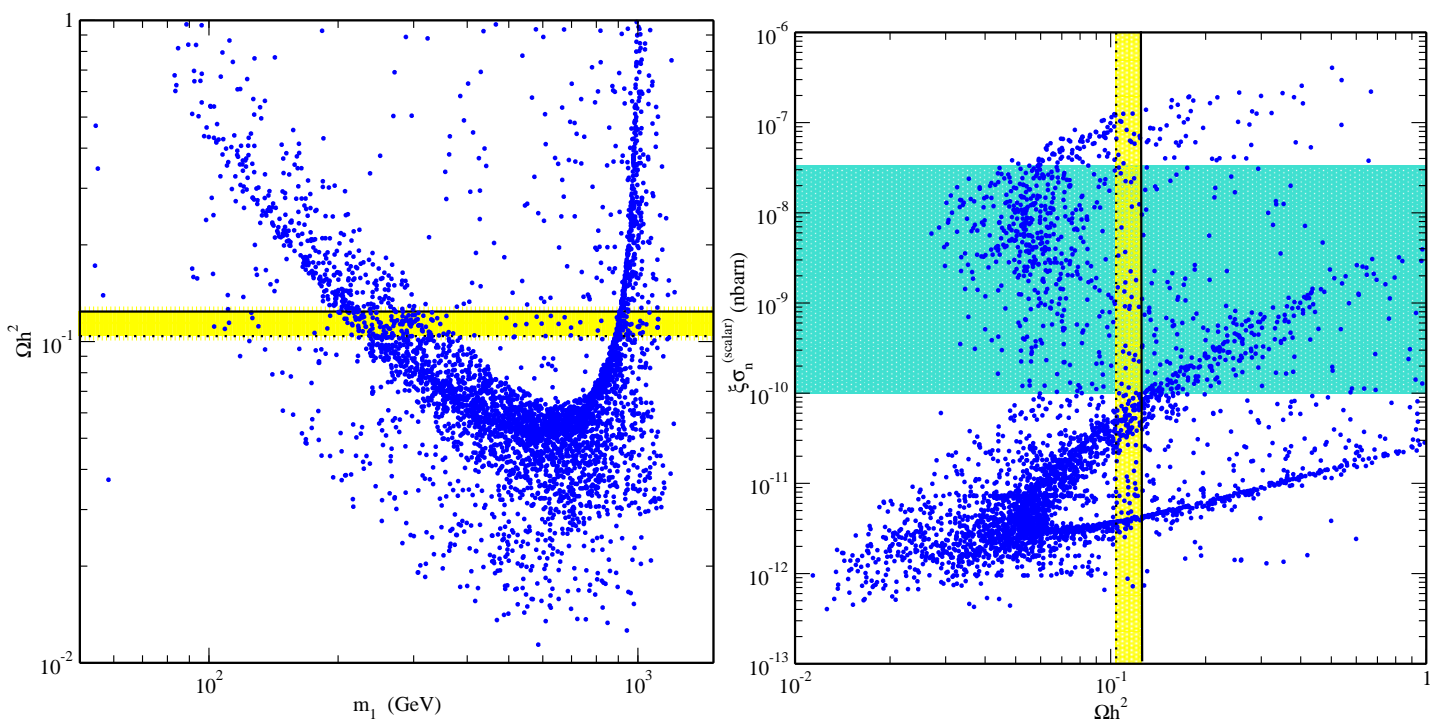

Figure 3: On the left panel sneutrino relic abundance $\Omega h^{2}$ as a function of the LSP sneutrino mass $m_{1}$, on the right panel sneutrino-nucleon scattering cross section $\xi \sigma_{\text {nucleon }}^{\text {(scalar) }}$ vs. the sneutrino relic abundance $\Omega h^{2}$, for a scan of the supersymmetric parameter space: $100 \mathrm{GeV}<m_{0}<3 \mathrm{TeV}, 100 \mathrm{GeV}<m_{1 / 2}<3 \mathrm{TeV}, A_{0}=0$, $3<\tan \beta<50,1 \mathrm{GeV}^{2}<B_{\mu_{S}}<80 \mathrm{GeV}^{2}, 500 \mathrm{GeV}<M_{R}<1 \mathrm{TeV}, 10^{-9} \mathrm{GeV}<\mu_{S}<10^{-6} \mathrm{GeV}$. The yellow band delimits the WMAP [8] cold dark matter interval at $3 \sigma$ of C.L.: $0.104 \leq \Omega_{\mathrm{CDM}} h^{2} \leq 0.124$.

The horizontal band denotes the current sensitivity of direct detection experiments; the vertical band delimits the $3 \sigma$ C.L. WMAP cold dark matter range [8].

neutrino masses and sneutrinos dark matter with the correct relic abundance indicated by WMAP as well as direct detection rates consistent with current dark matter searches.

\section{References}

[1] M. Taoso, G. Bertone and A. Masiero, JCAP 0803, 022 (2008) [arXiv:0711.4996 [astro-ph]].

[2] D.Cerdeno, C.Munoz, O.Seto, arXiv:0807.3029 [hep-ph].

[3] V. Berezinsky, J.F.W. Valle, Phys. Lett. B 318 (1993) 360; E. Akhmedov, Z. Berezhiani, R. Mohapatra, G. Senjanovic, Phys. Lett. B 299 (1993) 90; B. Mukhopadhyaya et al., Phys. Rev. D 76, 075001 (2007); M. Lattanzi, J.F.W. Valle, Phys. Rev. Lett. 99, 121301 (2007); F. Bazzocchi, M. Lattanzi, S. Riemer-Sorensen, J.F.W. Valle, arXiv:0805.2372.

[4] R.. Mohapatra, J.F.W. Valle, Phys. Rev. D34, 1642 (1986).

[5] F. Deppisch, J.F.W. Valle, Phys. Rev. D72, 036001 (2005).

[6] H. Nunokawa, S. Parke, J.F.W. Valle, Prog. Part. Nucl. Phys. 60, 338 (2008), this review gives an updated discussion of the standard seesaw mechanism and its variants.

[7] C. Arina, N. Fornengo, JHEP 11, 029 (2007).

[8] WMAP Collab., E. Komatsu et al., arXiv:0803.0547.

[9] W. Porod, Comput. Phys. Commun. 153, 275 (2003). 